\title{
Alessio Figalli: Magic, Method, Mission
}

Sebastià Xambó-Descamps (Universitat Politècnica de Catalunya (UPC), Barcelona, Catalonia, Spain)

This paper is based on [49], which chronicled for the Catalan mathematical community the Doctorate Honoris Causa conferred to Alessio Figalli by the UPC on 22nd November 2019, and also on [48], which focussed on the aspects of Figalli's scientific biography that seemed more appropriate for a society of applied mathematicians. Part of the Catalan notes were adapted to Spanish in [10]. It is a pleasure to acknowledge with gratitude the courtesy of the Societat Catalana de Matemàtiques (SCM), the Sociedad Española de Matemática Aplicada (SEMA) and the Real Sociedad Matemática Española (RSME) their permission to freely draw from those pieces for assembling this paper.

\section{Origins, childhood, youth, plenitude}

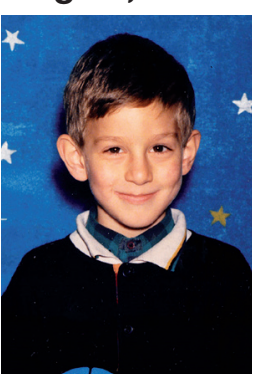

Alessio Figalli was born in Rome on 2 April 1984. His father Gennaro Figalli, now retired, was a professor of engineering, and his mother, Giuseppina Carola, is a teacher of Latin and Greek at a classical high school (liceo classico) in Rome. From Alessio's early years, in [45] Kevin Hartnett selects the following impressions:

As a kid Figalli liked to play soccer, watch cartoons, and hang out with his friends - and, he recalls, he always made the rational decision to get his homework done first, so that he could fully enjoy himself. "For me it was always a balance between how good a grade I could get and how much time I had to spend to get such a grade," he said. "I was always an optimizer, I wanted the best for the least effort."

In the interview [45], conducted by Helga Rietz, Figalli talks of his first experiences in relation to mathematics:

As a child I liked math because it was easy for me. I was thinking of being an engineer. Then I had to decide, at age 13, which high school I would like to attend. There are many types in Italy, but the main ones that prepare for the university are scientific and classical lyceums. In the latter you learn Greek and Latin, philosophy, etc., and I chose this option. In Italy there is always the idea that the classical high school offers the broadest education and that with this training you can later study whatever you want...

In his third year at high school, Antonio Corbo, a mathematician who worked at the same university as his father, suggested that Alessio participate in the Mathematical Olympiad. This led him to realise that there were mathematical problems whose solutions required inventive- ness, and his aptitude for solving them, as well as the joy such magic insights brought him, were a truly revelatory experience. In the aforementioned interview [45], Figalli describes this experience:

At the Mathematical Olympiad, I met other teens who loved math. All of them dreamed of studying at the Scuola Normale Superiore in Pisa (SNSP), which offers a high level of education. Those who get one of the coveted scholarships do not have to pay anything. Living, eating and studying are free. I also wanted that.

I concentrated on mathematics and physics on my own and managed to pass the entrance exam. The first year at Scuola Normale (SN) was tough. I didn't even know how to calculate a derivative, while my colleagues were much more advanced than me, since they came from the scientific lyceum.

He undertook a methodical study plan that allowed him to catch up with his peers within a year. How far he went is revealed by the following episode. At the beginning of his second year, he began to read a highly technical work that Luigi Ambrosio and Xavier Cabré had recently written [4]. Ambrosio expected that the novice student would have to persist for quite some time to make some progress. But the surprise for Ambrosio was, as stated in [42], that "Alessio came to see me less than a week later and I realised that he understood everything". This step marked Figalli's course in mathematical research: one year later he completed the bachelor's degree [22] and in the following three years he obtained the master's degree [23] and the doctorate [25], always with full honours. His doctoral thesis was supervised by Luigi Ambrosio and Cédric Villani (Fields Medal 2010). In the words of David Jerison (MIT), "Alessio is incredibly fast. Quick on the essentials and quick to isolate the important points".

Figalli's scientific output after his doctorate is surprising in all respects. Its leading role in the global mathematical landscape has steadily increased over the years. From the point of view of his academic positions, three phases can be distinguished. The first comprises of two terms: 2007-08, as a researcher at the University of Nice, and 2008-09, as Professeur Hadamard at the École Polytéchnique de Palaiseau. The second phase consists of the seven terms at the University of Texas at Austin (from 2009-10 to 2015-16): the first two as an associate professor (and Harrington Faculty Fellow in the first) and as professor in the following five (R. L. Moore Chair in the last three). Finally, since October 2016 he has been a professor at the ETH (Eidgenössische Technische Hochschule Zürich) and director of the Forschungsinstitut für Mathematik (FIM) since September 2019. 


\section{Rio de Janeiro, 1 August 2018}

It is the first time that the International Congress of Mathematicians (ICM) has been hosted in the southern hemisphere of the American continent. It was a milestone for Brazilian mathematics, in general, and for the Instituto Nacional de Matemática Pura e Aplicada (IMPA), in particular. In fact, its director, Marcelo Viana, was the president of the Congress.

It is also appropriate to remember that Jacob Palis had been president (1999-2002) of the IMU (International Mathematical Union) and that Artur Avila was awarded the Fields Medal at the Seoul ICM (2014) for his "profound contributions to the theory of dynamic systems". The highlight of the day, the most looked forward to, was the announcement of the Fields Medal recipients:

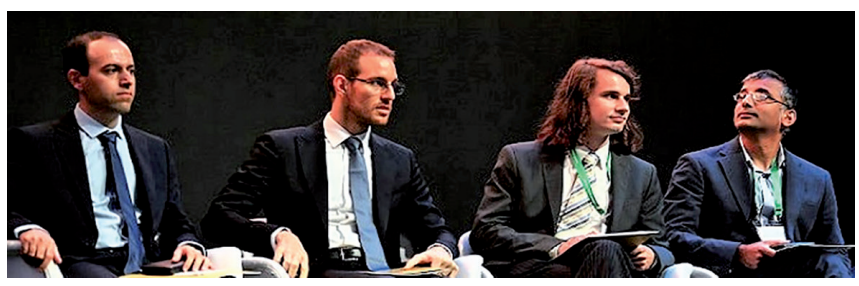

Caucher Birkar (Cambridge, UK), Alessio Figalli (ETH, Zürich), Peter Scholze (Universität Bonn) and Akshay Venkatesh (IAS, Princeton).

In the case of Alessio Figalli, the mention was for "contributions to the theory of optimal transport, and its applications to partial differential equations, to metric geometry and to probability theory". Luis Caffarelli, in charge of the laudatio, stated that "his work is of the highest quality in terms of originality, innovation and impact, both in mathematics itself and in its applications", and that he was destined "to be one of the most influential mathematicians of his generation".

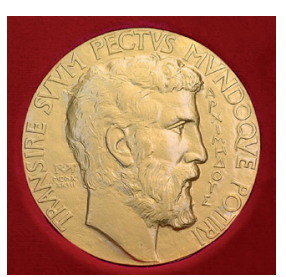

Figalli's plenary lecture as a medalist was titled "Regularity of interfaces in phase transitions via obstacle problems". Scheduled for Saturday, 4 August, it was a memorable occasion for its high quality, and also for the fact that at the end he mentioned recent results obtained in collaboration with Xavier Ros-Oton and Joaquim Serra. Both completed their studies in mathematics (bachelor and master) at UPC's Facultat de Matemàtiques i Estadística (FME), and earned a PhD from the UPC in June 2014 under the supervision of Xavier Cabré. They currently hold positions at the Universität Zürich (UZH) and the ETH, respectively. As stated on his website, Ros-Oton will hold positions at the "Institució Catalana de Recerca i Estudis Avançats" (ICREA), as a research professor, and at the Department of Mathematics of the Universitat de Barcelona, as a full professor, starting September 2020. It should also be mentioned that Serra has recently been awarded the EMS Prize.

\section{Scientific opus}

To begin with, it must be stated that an essential source for first-hand information about Alessio Figalli's life and work is his website at the ETH. Continuously updated, there one can find his curricula, in short and full versions; listings of his many awards, distinctions, honours and invited lectures; links to all his publications, classified according to different criteria; his commitments as editor of mathematical journals; lists of his doctoral students and postdocs; the many courses and seminars he has delivered; conference videos and links to interviews; and a special page for his ERC project. ${ }^{1}$ It is a huge and amazing work on all counts, particularly because at the moment of writing these notes (July 2020) the thirteenth anniversary of his doctoral thesis was still three months into the future.

In this section the main focus will be the section

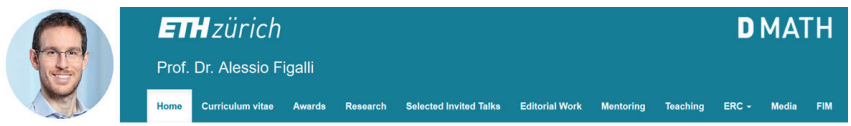

https://people.math.ethz.ch/ afigalli/.

"Research" of that web page, which is presented by declaring that his work falls within the areas of calculus of variations and partial differential equations (PDE),

with special emphasis on optimal transportation, Monge-Ampère equations, functional and geometric inequalities, elliptic PDEs of local and non-local type, free boundary problems, Hamilton-Jacobi equations, transport equations with rough vector-fields and random matrix theory.

The high quality of Figalli's research was masterfully outlined by Luis Caffarelli in his laudatio [18]:

A recurring theme in Figalli's research is the interaction between the theory of optimal transport and other areas of mathematics. Optimal transport theory, although pioneered by G. Monge back in 1781, has only transpired as being of paramount importance in many areas of mathematics in the past two decades. One of the most valuable features of this theory is its unifying power, in the sense that key ideas and constructions in optimal transport have turned out to be useful in the most unexpected contexts. Figalli is currently one of the most authoritative experts in optimal transport and its many applications.

Figalli's work is of the highest quality in terms of originality, innovation and impact both on mathematics per se as well as on its applications. He is clearly a driving force in the global mathematics community today. His approach to research is lively, dynamic and effective, and without a doubt will lead him to achieve many more stunning discoveries in years to come.

This last forecast is accurate if we are to judge by the quality and quantity of work produced in the last two years, and there is no doubt that it will continue at this or even a higher pace for years to come.

\footnotetext{
1 For a synopsis of a good part of this information, see https:// fme.upc.edu/ca/la-facultat/activitats/2019-2020/arxius/expo_ alessiofigalli-dhc.pdf, pp. 5 and 6.
} 


\section{Early productions}

We have already touched upon the beginnings of Figalli's mathematical career at the SNSP. Actually, the episode in Ambrosio's office, related to Figallis's reading of [4] (see also [1]), suggests a crystallisation of Figalli's mission with research in mathematical analysis at its core. The successive steps amply confirm this. At the end of the second year, he defends his bachelor thesis, which deals with topics related to the cited articles, and more specifically on the relations between the Bernstein problem and the De Giorgi conjecture [The terms or phrases in this font refer to the glossary at the end of this article; when they appear in quoted text, this emphasis is not on the original]. Two years later, at the end of his fourth term at SNSP, he defends his master's thesis. With surprising conceptual and technical maturity, he obtains, in particular, a comprehensive theory of optimal transport for noncompact Riemannian manifolds in this work. And a year later, at the age of twenty-three, he earns his PhD.

Let us take a moment to highlight some aspects of his doctoral thesis. As already stated, it was coadvised by Luigi Ambrosio and Cédric Villani. With more than 250 pages, each of its five chapters is based on one or more works already published or, at that time, pending publication. The titles of the chapters are quite vivid, but we find the description of their content in the thesis Introduction to be a bit more informative: 1) Optimal transport on manifolds with geometric costs (part of the results in collaboration with A. Fathi and C. Villani); 2) Optimal irrigation (with M. Bernot), a subject in which he quotes two pioneering works by Vicent Caselles [6,7]; 3) Brenier's variational theory of incompressible fluids (with L. Ambrosio); 4) Aubry-Mather theory and solutions of the Hamilton-Jacobi equations (with A. Fathi and L. Rifford); and 5) DiPerna-Lions theory on stochastic differential equation solutions (based on [26]). The thesis ends with an appendix with two sections, one dedicated to describing semi-concave functions and their properties with great generality, and another dedicated to Tonelli's Lagrangian functions, a theory that links the calculus of variations to the optimal transport. Finally, let us note that the paragraph that precedes these descriptions summarises the research philosophy of its author in two brushstrokes:

In the development of the theory of optimal transportation, as well as in the development of other theories, it is important on the one hand to explore new variants of the original problem, on the other hand to figure out, in this emerging variety of problems, some common (and sometimes unexpected) features. This kind of analysis is the main scope of our thesis.

By what has already been said, we notice Figalli's special predisposition towards sharing projects and research tasks with other people, generally leading the initiative. This observation is confirmed on verifying the large number of collaborators who participate in his publications, people who invariably have an outstanding profile. This is why it seems appropriate to document this aspect of his scientific record here. Basically, we will follow the order of the first research collaboration, as indicated by the year of the first article reviewed in Mathematical Reviews (MR), but grouped, for the convenience of the textual and graphic composition, in small successive groups (as in [40], poster 2).

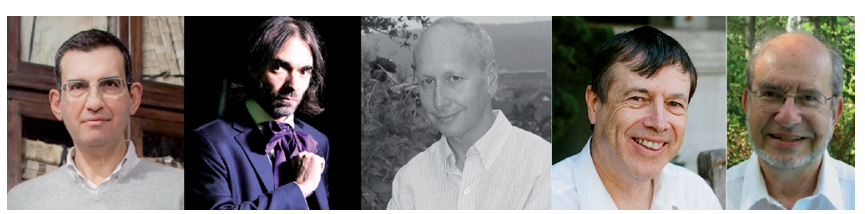

Luigi Ambrosio (SNSP. Fermat Prize 2003. Balzan Prize 2019); Cédric Villani (Institut Henri Poincaré, Université de la Sorbone, Université de Lyon, Institut Camille-Jordan. EMS Prize 2008. Fermat Prize 2009. Fields Medal 2010. Doob Prize 2014); Luis A. Caffarelli (University of Texas at Austin. Steele Prize 2009. Wolf Prize 2012. Shaw Prize 2018); Jean Bourgain (1954-2018. Fields Medal 1994. Shaw Prize 2010. Crafoord Prize 2012. Steele Prize 2018); Haïm Brezis (Université Pierre et Marie Curie. Distinguished Visiting Professor at Rutgers University. Author of the treatise [12] (English translation of his Analyse fonctionelle, 1983, it has been a constant reference textbook in many universities). According to ISI, he is a highly cited researcher).

This first group is headed by his thesis advisors (an exception to the ordering rule) and includes Luis A. Caffarelli (profiled at "ArbolMat", $\mathrm{AM}^{2}$ ), Jean Bourgain and Haïm Brezis. It should be noted, to avoid confusion, that our scheme does not take into account the number of collaborations, nor, in this group, the time at which they occurred. Thus, Bourgain and Brezis appear for a specific collaboration [3] (2016), announced in [2] (2014), which establishes a significant isoperimetric inequality related to a previous result of both authors (together with P. Mironescu: A new function space and applications). Luis Caffarelli is co-author of five papers, the first one in 2012 and the last one in 2020. The collaborations with Cédric Villani, six in total, occurred in the period 20072012, and with Luigi Ambrosio, fourteen, in the period 2008-2017.

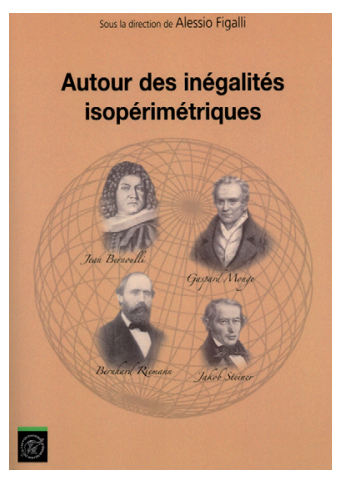

During his French period, he promotes the wonderful booklet Autour des inégalités isopérimétriques (Éditions de l'École Polytechnique, Palaiseau, 124 pages), which illustrates a productive model of collaboration, the interconnection of a diversity of subjects in the light of a common theme, and the nice coexistence of the research and didactic spirits. Figalli is the editor, and also the author of the preface. It is the outcome of the coordination, in the 2008-09 academic year, of a group of seven second-year students of the "École Politechnique" (W. Bench, C. De Franchis, L. Deproit, S. Gilles, B. Oh, A. Tenne, and K. Webster, who appear as authors) that "showed a particular interest in mathematics, and more specifically in the subject of isoperimetric inequalities,

2 RSME portal of Mathematics, Science and Technology, colloquially 'ArbolMat', or AM. 
which they wished to study both from a purely theoretical mathematical point of view and for their applications to other disciplines" (from the Preface). It contains seven chapters prepared jointly by the authors: 1) Relationship with the calculus of variations; 2) Generalization to any finite dimension; 3) Physico-chemical applications [shape of crystals, optimal structures; can you hear the shape of a drum?];4) Optimal transport;5) Algebraic generalization [contains a section on applications to graph theory]; 6) Generalization to manifolds; and 7) Conclusion [in which "some famous inequalities that could have led us to the isoperimetric inequality are mentioned"]. On the back cover we find a good description of the book's scope:

One of the oldest optimization problems is connected with isoperimetric inequalities. They are linked to many theories, the frameworks of which clarify and enrich each other. This work synthesizes these different theories, highlighting their interrelationships, and presents different applications of each of them in various fields. From the most abstract areas to questions of everyday life, this book shows how an apparently so simple and so specific inequality actually extends to entire branches of mathematics and other sciences.

In the second year of his stay in France, he lectures at the Bourbaki Seminar 2008-2009 on "Regularity of optimal transport maps (after Ma-Trudinger-Wang and Loeper)" [SB in what follows], which is published in Astérisque 332, 341-368, Société Mathématique de France (SMF). In addition, Villani's monumental work [47] on optimal transport appears and Figalli writes a detailed review [28].

These two works (and also [32]) are valuable indicators of the research context during his French stage and the degree of maturity and prestige that its author achieved in just seven years since entering the SNSP. In the acknowledgments, Villani writes that "Alessio Figalli took on the formidable challenge of reviewing the entire manuscript, from the first page to the last", with the connotation that he was the only one to accomplish such a feat. Naturally, this reading impacts on his review, whose purpose he sees as to "briefly examine the exciting and very active field of optimal transport, with an emphasis on the content and characteristics of the book being reviewed". In the review, Figalli also mentions [46], an interesting volume that in a way is a precursor to the book being reviewed, but which in fact has, by its approach, a complementary character.

The activity related to optimal transport is described authoritatively in the introductory paragraph of the review, as it appropriately corresponds to the leading status achieved by the reviewer since his doctoral thesis:

The problem of optimal transport has received the attention of many researchers in the past two decades, and its popularity continues to rise. This is mainly motivated by the discovery of unexpected connections between optimal transport and problems in physics, geometry, partial differential equations, etc.
He explains the optimal transport problem $(\$ 2)$ and the relationship of the optimal transport with the MongeAmpère equation (\$3). This section ends with the following comment:

In chapter 12, the author offers a very good introduction to the theory of regularity of optimal transport. But since the book was completed in 2008, some of the more recent developments linking the Ma-TrudingerWang tensor with the manifolds's geometry are not covered,

and refers to the SB article for a "recent presentation of these results". From the bibliography included in SB, it appears that among the results to which he refers, the following articles, of which he is co-author, should be counted: [33] (with Loeper), [35] (with Rifford), and [3638] (with Rifford and Villani, announced as "in preparation").

The article SB is also an introduction to the historical evolution of the subject, as it highlights some of the milestones achieved by the most outstanding researchers: Monge (1781), Brenier (1987, 1991), Caffarelli (1990, 1991, 1992, 1996), Urbas (1997), McCann (2001, 2007, 2008), Nirenberg (2005), Ma-Trudinger-Wang (2005), Liu (2009), Loeper (2009 - article in Acta Mathematica [43] -, 2011), Trudinger-Wang (2009), Loeper-Villani (2010), etc.

From the beginning of the term 2009-2010, Figalli's destination is the University of Texas in Austin, but in the academic year 2011-2012 he is once again connected with France on the occasion of being awarded the Peccot-Vimont Prize and in charge of the corresponding "Cours Peccot" at the "Collège de France": Stabilité dans les inégalités fonctionnelles, transport optimal et equations aux dérivées partielles. With this distinction he is in the company of illustrious predecessors, such as Laurent Schwartz (FM 1950), Jean-Pierre Serre (FM 1954), Alexander Grothendieck (FM 1966), Pierre Deligne (FM 1978), Alain Connes (FM 1982), Pierre-Louis Lions (FM 1994), Cédric Villani (FM 2010), or Artur Avila (FM 2014).

To these credentials we must add the Prize of the European Mathematical Society, which was awarded at the VI European Congress of Mathematics (2-6 July 2012, Kraków, Poland). The corresponding plenary lecture, entitled Stability in geometric and functional inequalities [29], describes, after honouring its title in detail, applications to the asymptotic behaviour of some evolution equations.

Let us now consider some of the most relevant results obtained in the 2009-10 to 2015-16 academic years, apart from those already mentioned. In addition to the original articles, the main references for consideration that follow are [18] (the Laudatio of Alessio Figalli by Luis Caffarelli at ICM2018, designated as [L] in what follows), and the database MathSciNet, designated as [MR].

Although Figalli's specialties are the PDE and the calculus of variations, it can also be asserted, with what we have already written, that in his research the devel- 


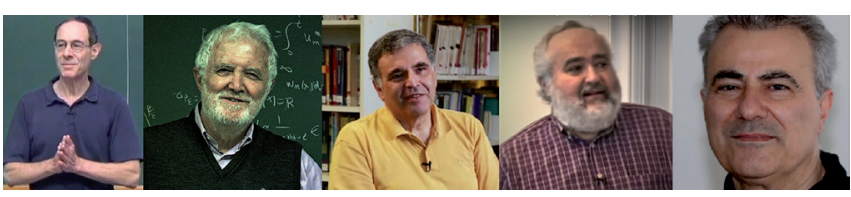

David Jerison (Massachusetts Institute of Technology. Bergman Prize 2012); Juan L. Vázquez (AM. Universidad Autónoma de Madrid. Premio Nacional de Investigación Julio Rey Pastor 2003. Plenary spekaer ICM-2006. Medal of the RSME 2018); Albert Fathi (Georgia Institute of Technology. Sophie Germain Prize 2013. Professeur émérite à l'ENS de Lyon); Thierry Paul (Directeur de Recherche, Centre de Mathématiques Laurent Schwartz, École Polytechnique, Paris, France); Henrik Shahgholian (Royal Institute of Technology (KTH), Stockholm, Sweden).

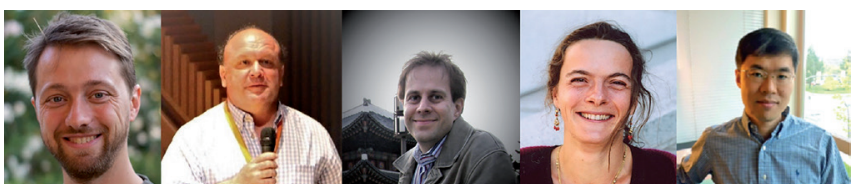

Francesco Maggi (University of Texas at Austin. Miranda Prize 2008); José A. Carrillo de la Plata (Imperial College London. Von Mises Prize 2006. Wolfson Prize 2012); Robert J. McCann (University of Toronto. Coxeter-James Prize 2005. Jeery-Williams Prize 2017); Alice Guionnet (École Normale Supérieure de Lyon. Loève Prize 2009. Pascal Medal 2018); Kim Young-Heon (University of British Columbia).

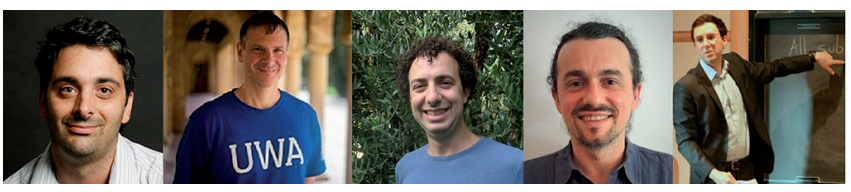

Ludovic Rifford (Université Nice Sophia Antipolis. Directeur du Centre International de Mathématiques Pures et Appliquées. Eisenbud Professor at MSRI 2013); Enrico Valdinoci (University of Western Australia. Most cited mathematician according to his graduation year (in all subjects and in Analysis). ERC Starting Grant 2011-2016); Aldo Pratelli (Università di Pisa. Medal of the President of the Italian Republic for young researchers 2004. Iapichino Prize 2005. ERC Starting Grant 2010-2015. Miranda Prize 2011. De Giorgi's Prize 2015); Matteo Bonforte (Universidad Autónoma de Madrid); Álvaro Pelayo (University of California at San Diego. Rubio de Francia Prize of the RSME 2009. NSF Faculty CAREER Award 2016).

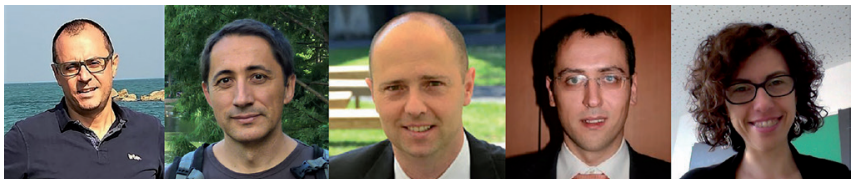

Marco Di Francesco (Dipartimento di Ingegneria e Scienze dell'Informazione e Matematica, Università degli Studi dell'Aquila); Dejan Slep̌cev (Department of Mathematical Sciences, Carnegie Mellon University); Giulio Ciraolo (Departimento di Matematica Federigo Enriques, Università degli Studi di Milano); Nicola Gigli (Scuola Internazionale Superiore di Studi Avanzati. De Giorgi's Prize 2019); Laura Valentina Spinolo (IMATI-CNR, Pavia, Italy).

opment of the theory of optimal transport is central for the study of a multiplicity of spaces and cost functions, as well as for their application to a surprising variety of areas of mathematics. The power of this unifying capacity is especially manifest in cases where problems in one area can be fruitfully investigated with techniques developed in others. We have previously seen examples of this transfer, as in his doctoral thesis, in the articles [27] and [28], or in the book [21]. The following articles offer oth- er paradigmatic cases (the names of the co-authors are included in parentheses after the year of publication):

2010 (F. Maggi and A. Pratelli) [34]

"[...] a sharp quantitative stability theorem for the Wulff inequality [...] a most important mathematical result in our understanding of surface tension driven phase transitions, as it relates the microscopic structure of a given surface tension energy density to the macroscopic shape of the liquid/crystal observed at equilibrium. [...] its sharp stability version allows one to describe [...] every low energy state. This is a result of clear physical importance, whose proof required several original and innovative mathematical ideas.] [L]

2012 "Stability in geometric and functional inequalities. Proceedings of the 6th ECM (Kraków, Poland, 2012), 585-599.

In thic congress, Alessio Figalli was awarded the EMS Prize,

For his outstanding contributions to the regularity theory of optimal transport maps, to quantitative geometric and functional inequalities and to partial solutions of the Mather and Mañé conjectures in the theory of dynamical systems.

2013 (G. De Philippis) " $W^{2,1}$ regularity for solutions of the Monge-Ampère equation". Inventiones mathematicae 192.1, 55-69.

"... a fundamental, groundbreaking result on the second order Sobolev regularity of optimal transport maps and their link with the Monge-Ampère equation" [L]. This result allowed the authors to understand the existence and regularity of solutions to the semigeostrophic equations, a classical system of partial differential equations used in meteorology.

2016 (A. Guionnet) "Universality in several-matrix models via approximate transport maps". Acta Mathematica 217.1, 81-176.

"Large random matrices arise as a natural model in diverse fields such as quantum mechanics, quantum chaos, telecommunications, finance and statistics. The central mathematical question in this area is how much their asymptotic properties depend on the fine details of the model. [...] Little was known about the universality of the fluctuations of the eigenvalues in severalmatrix models, except in very particular situations. Figalli [...] developed a new approach to these questions by introducing particular approximate transport maps. [...] This is a real breakthrough, which in addition gives a firm mathematical grounding to the widely held belief coming from physics that a universality of local fluctuations holds, at least until some phase transition occurs." [L]

2017 The Monge-Ampère equation and its applications [30]

By the year of its publication, this work would correspond to the Zürich stage, but the author tells us that 


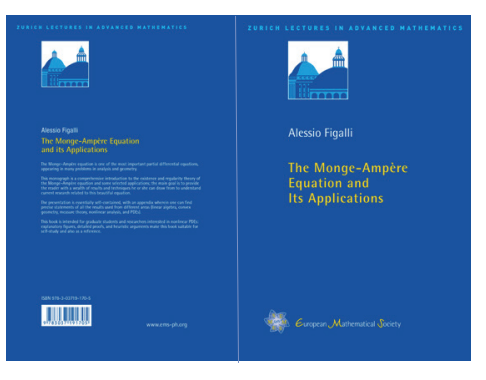

its origin is in "a series of lectures given by the author at ETH during the fall of 2014" with the same title. The purpose of the book is to provide a "a comprehensive introduction to the existence and regularity theory of the Monge-Ampère equation" and "to show some selected applications". His motivation, in contrast with the "classical" text [41], is "to include recent developments in the theory", fundamentally (Chapter 4) the study of the "interior regularity of weak solutions", "the geometry of solutions, mostly investigated by Caffarelli in the 1990s", and a proof of "interior $C^{1, \alpha}, W^{2, p}$ and $C^{2, \alpha}$ estimates". In addition, Chapter 5 presents "some extensions and generalisations of the results described in the previous chapters". The rest of the book is an extensive appendix (32 pages) in which the reader can find "precise statements of all the results used", and even, "whenever possible [...] proofs of such results". Topics covered are linear algebra, convex geometry, measure theory, nonlinear analysis and partial differential equations. The bibliography contains 125 entries, each with a link to the Zentralblatt review in all cases where it exists.

Now, before turning to Figalli's work in the ongoing ETH period (since 2016), let us display the two most recent rows in the gallery of Alessio Figalli's collaborators.

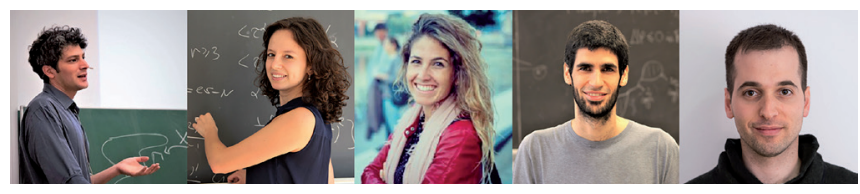

Guido De Philippis (Courant Institute, New York University. EMS Prize 2016. Stampacchia Medal 2018. Doctorate from the SNSP, supervised by L. Ambrosio and L. Caarelli); Maria Colombo (EPFL Lausanne. Iapichino Prize 2016. Miranda Prize 2018. Doctorate from the SNSP, supervised by L. Ambrosio and A. Figalli); Begoña Barrios (Universidad de La Laguna, Tenerife, Spain. Doctorate from the UAM, supervised by Ireneo Peral Alonso and Fernando Soria); Xavier Ros-Oton (Universität Zürich. Rubio de Francia Prize of the RSME 2016. Antonio Valle Prize of the SEMA 2017. ERC Starting Grant 2018. Fundación Princesa de Girona Prize 2019. Doctorate from the UPC, supervised by Xavier Cabré); Joaquim Serra (ETH Zürich. Évariste Galois Prize of the SCM 2011. Josep Teixidó Prize of the SCM 2016. Antonio Valle Prize of the SEMA 2019. EMS Prize 2020. Rubio de Francia Prize of the RSME 2019. Doctorate from the UPC, supervised by Xavier Cabré).

2017 (D. Jerison) "Quantitative stability for the BrunnMinkowski inequality". Advances in Mathematics 314, $1-47$.

"[...] succeeded in the quite challenging task of combining tools from additive combinatorics, affine geometry, harmonic analysis and optimal transport to obtain the first quantitative stability result for the BM inequality in arbitrary dimension and on generic sets. [...] quite impressive, both for the technical complexity, the wealth of original ideas involved, and the mathematical beauty of the question under consideration. [L]

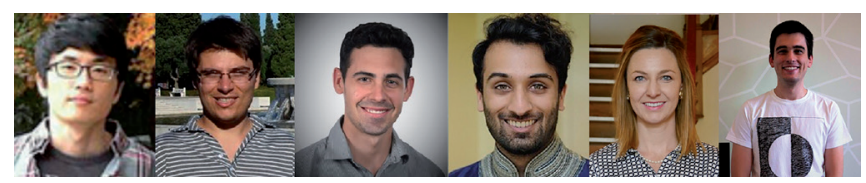

Moon-Jin Kang (SookmyungWomen's University, Seoul, South Korea. Doctorate from the Seoul National University, supervised by Seung Yeal Ha); Emanuel Indrei (Department of Mathematics, Purdue University. Doctorate by the University of Texas at Austin, supervised by A. Figalli); Connor Mooney (University of California at Irvine. Firestone Medal for Excellence in Undergraduate Research, Stanford University, 2011. Doctorate from Columbia University, supervised by Ovidiu Savin); Yash Jhaveri (Princeton Institute for Advanced Study. Doctorate from the ETH Zürich, supervised by A. Figalli); Robin Neumayer (Northwestern University. Doctorate from the University of Texas at Austin, supervised by A. Figalli and F. Maggi); Xavier Fernández-Real (PhD student, supervised by A. Figalli at ETH Zürich. Whelan Prize in Mathematics from Christ's College, University of Cambridge, 2015. Évariste Galois Prize of the SCM, 2016. Ranked First in the Spanish National Award for Excellence in Academic Record 2013-2014, 2018. Coauthor, with X. Ros-Oton, of the memoir Regularity Theory for Elliptic PDE (2020). ${ }^{3}$

2017 (M. Cozzi) "Regularity Theory for Local and Nonlocal Minimal Surfaces: An Overview". Nonlocal and Nonlinear Diffusions and Interactions. New Methods and Directions (Cetraro, 4-8 julio, 2016), Lecture Notes in Mathematics 2186, 117-158. CIME Foundation Subseries, Springer (edited by Matteo Bonforte and Gabrielle Grillo).

This article is a basic reference on the subject of minimal surfaces. The volume also contains articles by José Antonio Carrillo (with Vincent Calvez and Franca Hoffmann), Manuel del Pino, Giuseppe Mingione and Juan Luis Vázquez (whose seventieth anniversary was celebrated with a special dinner in which his "big involvement in Italian mathematics" was highlighted).

2019 (J. Serra) "On the fine structure of the free boundary for the classical obstacle problem". Inventiones mathematicae 215.1, 311-366.

"[...] great acclaim greeted the work of Figalli, together with his co-author Joaquim Serra, who in 2017 gave a complete and definitive description of the free boundary. [...] The new methods introduced in this work are having a wide impact. (A. Jackson, The Work of Alessio Figalli, IMU, 2018).

2020 (J. Serra) "On stable solutions for boundary reactions: a De Giorgi-type result in dimension 4+1". Inventiones mathematicae 219.1, 153-177.

\section{0 (X. Cabré, X. Ros-Oton, J. Serra) [16]}

"[...] we prove the following long-standing conjecture: stable solutions to semi-linear elliptic equations are bounded (and thus smooth) in dimension $n \leq 9$. This result, that was only known for $n \leq 4$, is optimal: $\log \left(1 /|x|^{2}\right)$ is a $W^{1,2}$ singular stable solution for $n \geq 10$. [...] As one can see by a series of classical examples, all our results are sharp. Furthermore, as a corollary,

\footnotetext{
3 https://people.math.ethz.ch/ xavierfe/Documents/FernandezReal_Ros-Oton_Book.pdf
} 
we obtain that extremal solutions of Gelfand problems are $W^{1,2}$ in every dimension and they are smooth in dimension $n \leq 9$. This answers two famous open problems posed by H. Brezis and Brezis-Vázquez (from the Abstract; cf. [14], [13] and [11]).

\section{0 (Ros-Oton, J. Serra) [39]}

\section{UPC Doctorate Honoris Causa}

Alessio Figalli was conferred a Doctorate Honoris Causa by the UPC on Friday, 22 November 2019, with Xavier Cabré in charge of the laudatio. ${ }^{4}$

The day before, on 21 November 2019, the FME organised a workshop in homage of the honoree. The invited speakers are outstanding collaborators of Figalli's: Juan Luis Vázquez and Matteo Bonforte, from the UAM; Ros-Oton and Serra, already mentioned, of the younger generation; and Cabré, who is responsible for closing the day. 5

The panel is scheduled as the first activity in the afternoon, just before the lectures by Serra and Cabré. The panel members are Figalli, the five lecturers of the day, and the Dean (Jaume Franch), who chaired the activity. The game is to ask a round of questions to Figalli with the purpose of discovering aspects of his trajectory, experience and thinking that can hardly be elicited from his mathematical writings.

The first move is by Juan Luis Vázquez. After acknowledging the great popularity of mathematics, largely derived from its many and attractive applications, he expresses some concern about the possible relaxation of content that this phenomenon could entail, and concludes by asking Figalli's opinion on these perceptions, and in particular about what balance should be achieved

Xavier Cabré has been an ICREA research professor since 2003 at the UPC's Department of Mathematics (DMAT), and full professor of applied mathematics since 2008. He earned his doctorate in 1994 at the Courant Institute in New York under the supervision of Louis Nirenberg (1925-2020) with the thesis Estimates for Solutions of Elliptic and Parabolic Equations. New York University Kurt Friedrichs Award, 1995. IAS Member, 1994-95. Habilitation à diriger des recherches, Université Pierre et

Marie Curie-Paris VI, 1998. Harrington

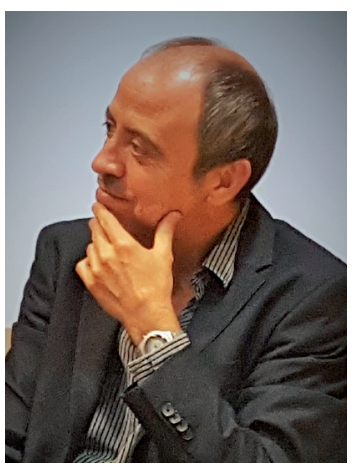

Faculty Fellow, The University of Texas at Austin, 2001-02. Fellow of the American Mathematical Society, inaugural class, 2012. Co-author, with Caffarelli, of the memoir [17]. As we have seen, the papers [4,1] were among the first research articles to influence Figalli. Moreover, his former doctoral students Xavier Ros-Oton and Joaquim Serra, and Cabré himself, count among Figalli's most important collaborators in recent years. His profile is included in AM.

4 Cabré's laudatio and Figalli's lectio honoris causa can be accessed at the UPC website: see [15, 31].

5 The corresponding presentations, including their videos, can be found at https://fme.upc.edu/ca/la-facultat/activitats/2019-2020/ Workshop-Figalli-FME-2019-ponencies-fotos-gravacio

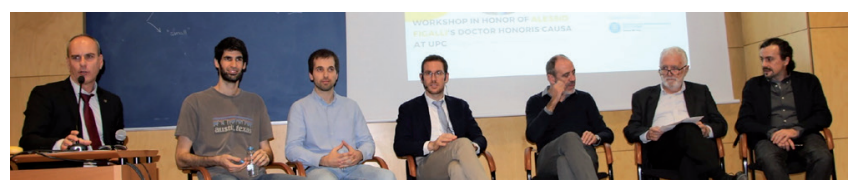

Jaume Franch, Xavier Ros-Oton, Joaquim Serra, Alessio Figalli, Xavier Cabré, Juan Luis Vázquez, Matteo Bonforte.

between the study of fundamental and applied mathematics in the bachelor's curricula.

In his response, Figalli states that he does not see a real dichotomy and provides several considerations in favour of this point of view. He argues that in some countries, especially the United States of America and the United Kingdom, it is possible to pursue very intensive studies of mathematics, very theoretical, which give one the option of deciding later whether to continue in the academic sector or move to other areas in which their profiles are highly appreciated. This approach provides attractive opportunities for students who would otherwise go directly to careers where it is initially easier to find a job. In fact, this also favours that more people have the opportunity to dedicate themselves to mathematics.

Xavier Cabré asks Figalli if he can briefly describe the role of the SNSP in the Italian university system, also how important it was for his own career, and finally whether he can share any suggestions on how Spain could maximize the chances of getting a Fields Medal in the future.

"Good question! Yes, it is fortunate for Italians to have the SNSP, a French-like institution founded by Napoleon", says Figalli, adding that it was excellent for $\mathrm{him}$. The entrance is competitive, open to all, and during the studies the system covers all expenses. He values as very positive that the state promotes equal opportunities and in that line welcomes the more recent creation of other research institutions, such as the Scuola Internazionale Superiore di Studi Avanzati (SISSA), in Trieste, and the Istituto Universitario di Studi Superiori (IUSS), in Pavia. Regarding the access to important distinctions and awards, Figalli thinks that the possibilities are correlated with the number of people in a community who acquire a robust mathematical training, and that the visits to prestigious centres around the world are very important, both for the Fields Medal and for many other awards.

Xavier Cabré asks what differences there are between studying at the SNSP or at another Italian university. The answer is that the study regime at the SNSP has a dual character, since it is based on being enrolled at the University of Pisa, like any other student of this institution, and supplementing it with additional courses at the SNSP, such as analysis and physics in the first year; measure theory, probability and electromagnetism in the second; and algebraic geometry and quantum mechanics in the third. For the fourth and fifth years, he does not specify any discipline, since they can vary from one year to another. Another distinctive feature he mentions is the problem list assigned every week. Indeed, each student is responsible for presenting a detailed written solution to one of the problems and sharing it with the class, a setup 
that favours the development of the spirit and skills that a researcher needs. He considers that their solutions help to understand more fully the meaning of mathematics beyond the abstract theorems learned in theory.

In the next intervention, Matteo Bonforte is concerned with the proportion of research time to be devoted to problems which are considered difficult, and also with whether to worry, at any level (from students to established researchers), if the solution is not found immediately.

From the outset, Figalli thinks that it would be fallacious to give an answer for everyone, since the differences between people are considerable. There are some that are very effective at concentrating on a single problem for a long time, such as Andrew Wiles with Fermat's theorem. Others work with a more functional orientation, in the sense that they focus on a certain group of problems, which favours a more distributed and staggered treatment of difficulties. In collaborative work, he suggests bearing in mind that progress is often reinforced by differences in style rather than coincidences.

In the case of students, particularly doctoral students, he thinks that the distribution of effort is an issue in which supervision should be essential. Once reasonable research autonomy has been achieved, each person must find out what best suits them. As an illustration, he explains his work on the Monge-Ampère equations, in collaboration with De Philippis, which started in 2005 and was not completed until 2012. Initial attempts lasted months, but were unsuccessful. When they finally resumed the research, they found that the key ideas did not emerge until quite late. An important final consideration is that in his case he learned many things by struggling with problems in which repeated attempts were unsuccessful.

Xavier Ros-Oton puts forward the question of contrasting the merits and demerits of two ways of evaluating research: that of countries such as Spain and Italy, in which published articles or citations are counted, and that of countries such as the United States, Germany or Switzerland, in which the main evidences stem from the recommendation letters by experts and the quality of the best research carried out.

After considering that the issue is very delicate, Figalli first describes how the Italian system works, then assesses the role of the letters of recommendation and, finally, defends a supervised system, based on clear rules of procedure, that can guarantee the highest objectivity and transparency. As for the Italian system, the averaging filter stands out. To be considered for a habilitation, for example, one must have a number of publications not less than that reached by $60 \%$ of the researchers in the area, and similarly with the number of citations and with the so-called index $h$. Among the problems of this system, he points out that it can leave out important papers, also the absence of a minimum quality control of those that exceed the indicators, and the fact that the definition of the averages implies that it is increasingly difficult to reach them.

The justification for the letters of recommendation is that there comes a time when you have to enter the contents and their significance, assess their real impact regardless of the journal in which they have been published, since no magazine can be an indisputable reference for all areas, and for that purpose an appropriate selection of experts can be decisive. But it also warns that it cannot be abused, since it involves a lot of effort on everyone's part, so its use is only recommended after some type of prior filter.

Finally, Figalli expresses that he likes the rules-based systems, for example to deal with conflicts of interest, as commonly used in the USA. A system of clear, impersonal rules simplifies decision-making, especially if the process is supervised, as is the case in Switzerland, by someone who knows how to guide its unfolding regardless of the people involved. A supervisor can ask a person to leave the room, after hearing what he or she wants to say, if that person has a conflict of interest in the matter to be resolved. Such a decision would hardly work spontaneously if the request had to come from one of the committee members.

During his turn, Joaquim Serra introduces the topic of rating the professional destinations of the doctorates in mathematics. While in countries like the USA and Switzerland the majority go into industry, and that these destinations are well regarded, in others, such as Italy and Spain, it is perceived as a certain devaluation of the academic career, although this impression may have begun to change already. How could the university benefit from the prestige of mathematics in society if it increased the number of excellent doctorates in non-academic positions and, therefore, the university's interaction with society? In his answer, Figalli ponders that the difficulty lies, at least in the case of Italy, in the fact that for companies the doctoral degree does not usually represent any added value compared to a master's degree. It is natural, therefore, that potential doctoral students do not see, from the point of view of jobs in the business world, any tangible benefit in the effort to obtain a doctorate. In the USA, on the other hand, a doctorate is well considered, a fact that is reflected, as a general rule, in better wages. However, the social benefit of the doctorate lies in the people trained to think critically, to solve problems, skills that are relevant for many occupations. As the acceptance of this view by society is an upward value, so is the benefit obtained by the university. Achieving a mature university-business interaction has in its favour the fact that the number of higher graduates in mathematics is very small compared to the population of all graduates of similar levels. Figalli also considers some of the elements of the situation at ETH that enhance this dynamic. The polytechnic nature of ETH implies that the Department of Mathematics teaches in all other departments. This guarantees a good mathematical foundation for all, leading to a better acceptance of the role of mathematicians in industry, which in turn allows the Department of Mathematics to access more resources, particularly for research. This circle indicates that the industry can be an ally, and as an illustration mentions the joint doctorates with companies such as Google in the case of ETH. This is a good time for maths! 
The last question from the panel is asked by Jaume Franch, in two parts: For a student who has just received a doctorate, which strategy is better: to stay in the same institution and try to get a job at it or venture to go abroad? In this sense, how important has your stay in Austin been for your fulfillment?

The predictable answer is clear: Figalli is personally against staying in the same place, but it seems natural to him that there are people who return later. In fact, he thinks that a sojourn of a few years abroad is a very positive experience for everyone, more than anything because of the gain in knowledge and experience. His own training as a mathematician began in Italy, where he learned the techniques of the De Giorgi school, including calculus of variations and geometric measure theory. He then spent three years in France, where he became familiar with the interrelationship between optimal transport and isoperimetric inequalities, and finally came his stay in Austin, hosted by Luis Caffarelli, and where he solved, after three years, the problem of regularity for the Monge-Ampère equation (solution already alluded to above, see p. 22). He underlines that the climate of Austin was very important for the kind of research he was working on in that period. In fact, he stresses that he considers himself a product of his travels and thinks that this recipe may be valuable for other researchers.

Taking advantage of the opportunity given by the Dean to the assistants, a doctoral student states that he very much values research collaborations, as he sees in them many advantages, but with the disadvantage,

\section{Glossary}

If $X \subset \mathbb{R}^{n}$ is measurable, $|X|$ denotes the Lebesgue measure of $X$.

Bernstein problem. A function $f: \mathbb{R}^{n-1} \rightarrow \mathbb{R}$ whose graph in $\mathbb{R}^{n}$ is a minimal hypersurface (that is, such that it satisfies $\operatorname{div}\left(\nabla f / \sqrt{1+|\nabla f|^{2}}\right)=0$, which is the Euler-Lagrange equation deduced from the area functional $\left.A(f)=\int \sqrt{1+|\nabla f|^{2}}\right)$, is it necessarily linear? In 1914, Sergei N. Bernstein (18801968) solved it affirmatively for $n=3$. See [9], in which the question is answered affirmatively for $n \leq 8$ and negatively for $n \geq 9$. For a report on the successive advances on this matter, including its close relationship with the De Giorgi conjecture, see [1].

Brenier theorem. Let $X$ and $X^{\prime}$ be open sets in $\mathbb{R}^{n}$ with probability distributions $\mu$ and $\mu^{\prime}$, respectively. Let $c: X \times$ $X^{\prime} \rightarrow \mathbb{R}$ be the cost function $c\left(x, x^{\prime}\right)=\left|x-x^{\prime}\right|^{2}$ and assume that that $\mu$ is absolutely continuous with respect to the Lebesgue measure. Then there exists a unique optimal transport $T$ from $\mu$ to $\mu^{\prime}$. Moreover, there is a convex function $u: X \rightarrow \mathbb{R}$ such that $T(x)=\nabla u(x)$ for all $x \in X$. If in addition $\mu=f d x, \mu^{\prime}=f^{\prime} d x$ and $T$ is a diffeomorphism, then $u$ satisfies $\operatorname{det}\left(D^{2} u\right)=f / f^{\prime}(\nabla u)$, which is a Monge-Ampère equation. See [5].

Brunn-Minkowski inequality. Given two compact sets $X$ and $Y$ in $\mathbb{R}^{n}$, and setting $X+Y=\{x+y: x \in X, y \in Y\}$ according to people in his environment, that the credit for the work is diluted.

In Figalli's comment, he first points out that there are many differences between people in this regard, and that the views of the thesis advisor can be decisive. He also considers that some publication should be about a problem solved from beginning to end by the student, since this autonomy is part of what the access to a doctor's degree should insure. On the other hand, what mainly counts in the publications are the results, whether in collaboration or not. To illustrate this, he comments on his first paper. At the beginning of his doctorate, his supervisor, Luigi Ambrosio, sent him to Lyon for a semester. There he met Albert Fathi, who gave him a recent article on optimal transport on compact manifolds, and suggested that it would be nice to extend it for non-compact manifolds. Figalli reacted by saying that it seemed very easy, which led Fathi to say that precisely because of that it was an interesting problem to start with. Figalli went to work and realised that the generalisation had more difficulties than he had originally foreseen. Filling in all the details, and writing them in an orderly and complete manner, was a decisive learning process at that point in his career [24].

Xavier Cabré supplements the previous comments with the idea that one encounters difficult problems that can probably only be solved by collaborating with other researchers, and states his belief that this awareness has a positive effect on the research dynamics.

(the Minkowski sum of $X$ and $Y$ ), then $|X+Y|^{1 / n} \geq|X|^{1 / n}+$ $|Y|^{1 / n}$. See $[46, \S 6.1 .2]$ for an elementary proof using mass transportation theory. Intuitively, the "linear size" of $X+Y$ is at least the sum of the "linear sizes" of $X$ and $Y$.

De Giorgi conjecture. Let $u: \mathbb{R}^{n} \rightarrow(-1,1)$ be a $C^{2}$ solution of the semi-linear equation $\Delta u=u^{3}-u$ such that $\partial_{x_{n}} u>0$ in $\mathbb{R}^{n}$. Then all level sets $\{u=s\}$ are hyperplanes, at least for $n \leq 8$. See [1].

geometric costs. They are costs $c\left(x, x^{\prime}\right)$ defined on a manifold $X$ given by expressions of the form

$$
c\left(x, x^{\prime}\right)=\inf _{\gamma} \int_{0}^{1} L(\gamma, \dot{\gamma}) d t,
$$

where $L$ is a Tonelli Lagrangian (see [24, §6.2]) and the infimum is taken with respect to all absolutely continuous curves on $X$ going from $x$ to $x^{\prime}$.

isoperimetric inequality. If $X \subset \mathbb{R}^{n}$ is regular and bounded, $|\partial X| \geq n|B|^{1 / n}|X|^{(n-1) / n}$, where $B \subset \mathbb{R}^{n}$ denotes the ball of radius 1. See [30, §4.6.2] for a brief proof using optimal transport. This inequality is stable, in the sense that if $X$ almost reaches equality, then $X$ is quantitatively close to a ball: See [34].

Minkowski problem. Let $X \subset \mathbb{R}^{n+1}$ be the graph of a function $u: \Omega \rightarrow \mathbb{R}, \Omega \subseteq \mathbb{R}^{n}$ open, and let $K: \Omega \rightarrow \mathbb{R}$. The condition for $X$ to have curvature $K(x)$ at $(x, u(x))$ turns out to be a Monge-Ampère equation: $\operatorname{det}\left(D^{2} u\right)=K\left(1+|\nabla u|^{2}\right)^{(n+2) / 2}$. 
Monge-Ampère equation. Given a function $u: \Omega \rightarrow \mathbb{R}$, $\Omega \subseteq \mathbb{R}^{n}$ open, it is an equation of the form $\operatorname{det}\left(D^{2} u\right)=f$, where $D^{2} u$ the Hessian of $u$ and $f=f(x, u, \nabla u)>0$ in $\Omega$. It is a nonlinear second order PDE.

optimal transport, $\sim$ map, $\sim$ problem. The optimal transportation problem is finding the cheapest way to transport a mass distribution from one place to another given some measure of cost. Seen in this way, it can be considered as a problem in economics, but it actually turns out to be a useful technique in PDE, fluid mechanics, geometry, probabilities or functional analysis. The variability of this scheme is unlimited in terms of the geometry of the ambient space and the shape of the cost function, and in each case the basic questions are to ensure the existence, uniqueness and regularity of optimal transport.

Formally, and with the notations introduced in transport map, let $c: X \times X^{\prime} \rightarrow \mathbb{R}^{+}$(cost function). A transport $T:$ $X \rightarrow X^{\prime}$ from $\mu$ to $\mu^{\prime}$ is optimal if the total cost of $T$, which by definition is $\int_{X} c(x, T(x)) d \mu(x)$, is minimal with respect to all transports from $\mu$ to $\mu^{\prime}$. In the case where $X^{\prime}=X$ and $d$ is a distance on $X$, then $d^{p}\left(x, x^{\prime}\right)(p>0)$ is an example of a cost function. The case $p=2$ is of special importance, particularly when $X$ is an open set of $\mathbb{R}^{n}, \mu=\rho d x$ and $\mu^{\prime}=\rho^{\prime} d x$ ( $\rho$ and $\rho^{\prime}$ positive functions with compact support) and $d$ the Euclidean distance, since these conditions guarantee the existence of a single optimal transport and that it has the form $T=\nabla u, u$ a convex function [5]. Cf. Brenier theorem.

semigeostrophic equations. In the case of the atmosphere, the geostrophic flow assumes that the pressure gradient is in equilibrium with the Coriolis force (geostrophic balance), in which case the wind direction is parallel to the isobars.

\section{References}

[1] Giovanni Alberti, Luigi Ambrosio, and Xavier Cabré. On a longstanding conjecture of E. De Giorgi: Symmetry in 3D for general nonlinearities and a local minimality property. Acta Applicandae Mathematica, 65(1-3):9-33, 2001.

[2] Luigi Ambrosio, Jean Bourgain, Haïm Brezis, and Alessio Figalli. Perimeter of sets and BMO-type norms. CRASP, Série I, 352(9): 697- 698, 2014

[3] Luigi Ambrosio, Jean Bourgain, Haïm Brezis, and Alessio Figalli. BMO-type norms related to the perimeter of sets. Communications on Pure and Applied Mathematics, 69(6):1062-1086, 2016.

[4] Luigi Ambrosio and Xavier Cabré. Entire solutions of semilinear elliptic equations in R3 and a conjecture of De Giorgi. Journal of the American Mathematical Society, 13(4):725-739, 2000.

[5] Jean-David Benamou and Yann Brenier. A numerical method for the optimal time-continuous mass transport problem and related problems. Contemporary mathematics, 226:1-12, 1999. In volume [19].

[6] Marc Bernot, Vicent Caselles, and Jean-Michel Morel. Traffic plans. Publicacions Matemàtiques, pp. 417-451, 2005.

[7] Marc Bernot, Vicent Caselles, and Jean-Michel Morel. The structure of branched transportation networks. Calculus of Variations and Partial Differential Equations, 32(3):279-317, 2008.

[8] Xavier Blanc and Mathieu Lewin. The crystallization conjecture: a review, 2015. https://arxiv.org/pdf/1504.01153.pdf.

[9] Enrico Bombieri, Ennio De Giorgi, and Enrico Giusti. Minimal cones and the Bernstein problem. Inventiones Mathematicae, 7:243-268, 1969.
In semi-geostrophic flow, this balance occurs only approximately and other forces are taken into account. One of the important equations of this flow is in the form of a MongeAmpère equation, which points out to the role of this equation in such dynamics.

transport map. Let $\mu$ and $\mu^{\prime}$ be probability distributions defined over measurable spaces $X$ and $X^{\prime}$, respectively. A measurable map $T: X \rightarrow X^{\prime}$ is said to transport $\mu$ in $\mu^{\prime}$ if $T_{\sharp} \mu=\mu^{\prime}$, that is, if $\mu\left(T^{-1} A^{\prime}\right)=\mu^{\prime}\left(A^{\prime}\right)$ for all measurable $A^{\prime} \subseteq X^{\prime}$.

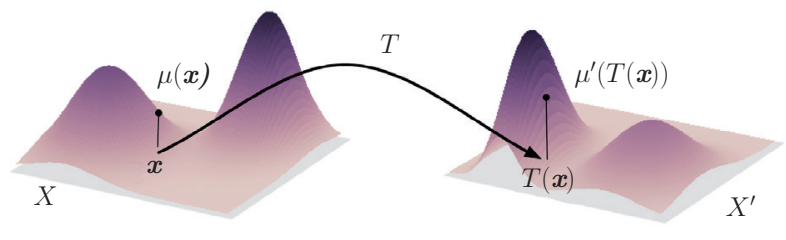

Wulff inequality. It appears in the study of crystals and phase transitions. On convex sets, it is equivalent to BrunnMinlowski inequality. In [34] the optimal transport technique is used to establish an improved inequality for convex sets $X, Y \subset \mathbb{R}^{n}:|X+Y|^{1 / n} \geq\left(|X|^{1 / n}+|Y|^{1 / n}\right)\left(1+c_{n}(A(X, Y))\right)$, with $c_{n}$ a constant that only depends on $n$ and $A(X, Y)$, a function that measures the "relative skewness" between $X$ and $Y$.

Wulff shape, Wulff construction. It is the equilibrium surface for a crystal or a drop that has minimal anisotropic surface energy for a given volume. In the isotropic case it is the sphere. See [20, Ch. 5], [44, Ch. 7], [8]. The Wulff construction is a method for finding the equilibrium shape (Wulff shape) of a drop or crystal of a given volume.

[10] Matteo Bonforte and Sebastià Xambó-Descamps. Alessio Figalli. La Gaceta de la RSME, 23(2):405-434, 2020.

[11] Haïm Brezis. Is there failure of the inverse function theorem?, 1999. Proceedings of the Workshop held at the Morningside Center of Mathematics, Chinese Academy of Science, Beijing, June 1999. Published in the volume Morse theory, minimax theory and their applications to nonlinear differential equations, New Studies in Advanced Mathematics, 1, International Press, Somerville, MA, 2003, 23-33.

[12] Haïm Brezis. Functional analysis, Sobolev spaces and partial differential equations. Springer, 2010.

[13] Haïm Brezis and Xavier Cabré. Some simple nonlinear PDE's without solutions. Bollettino della Unione Matematica Italiana-B, 1(2):223-262, 1998.

[14] Haïm Brezis and Juan Luis Vázquez. Blow-up solutions of some nonlinear elliptic problems. Revista Matemática de la UCM,10(2): 443- 469, 1997.

[15] Xavier Cabré. Laudatio HC, 2019. https://www.upc.edu/ca/la-upc/ discursos-honoris/laudatio-discurs-figalli-eng.pdf, p. 1-5.

[16] Xavier Cabre, Alessio Figalli, Xavier Ros-Oton, and Joaquim Serra. Stable solutions to semilinear elliptic equations are smooth up to dimension 9. Acta Mathematica, pages 187-252, 2020. https:// arxiv.org/ pdf/1907.09403.pdf.

[17] L.A. Caffarelli and X. Cabré. Fully nonlinear elliptic equations, volume 43 of AMS Colloquium Publications. American Mathematical Society, 1995.

[18] Luis Caffarelli. Alessio Figalli: His Contributions to Geometry 
and Analysis. In Boyan Sirakov, Paulo Ney de Souza, and Marcelo Viana, editors, Proceedings of the International Congress of Mathematicians (Rio de Janeiro, 1-9 August, 2018), Volume I, pages 43-46. World Scientific, 2018.

[19] Luis A. Caffarelli and Mario Milman (eds). Monge-Ampère Equation: Applications to Geometry and Optimization, volume 226 of Contemporary Mathematics. American Mathematical Society, 1999. NSF-CBMS Conference on the Monge Ampère Equation, Applications to Geometry and Optimization, July 9-13, 1997, Florida Atlantic University.

[20] Raphaël Cerf. The Wulff Crystal in Ising and Percolation Models. Springer, 2006. Ecole d'Eté de Probabilités de Saint-Flour XXXIV 2004 (Jean Picard, ed.).

[21] Alessio Figalli (ed.). Autour des inégalités isopérimétriques. Éditions de l'École polytechnique, 2011. Authors' pool, supervised by Alessio Figalli: W. Bench, C. De Franchis, L. Deproit, S. Gilles, B. Oh, A. Tenne and K. Webster.

[22] Alessio Figalli. Il problema de Bernstein e una congettura di De Giorgi, 2004. Bacherlor thesis, Università degli Studi di Pisa.

[23] Alessio Figalli. Trasporto ottimale su varietà non compactte, 2006. Master thesis, Università degli Studi di Pisa.

[24] Alessio Figalli. Existence, uniqueness, and regularity of optimal transport maps. SIAM journal on mathematical analysis, 39(1):126-137, 2007.

[25] Alessio Figalli. Optimal transportation and action-minimizing measures. PhD thesis, Scuola Normale Superiore of Pisa and École Normale Supérieure of Lyon, 2007.

[26] Alessio Figalli. Existence and uniqueness of martingale solutions for SDEs with rough or degenerate coefficients. Journal of Functional Analysis, 254(1):109-153, 2008

[27] Alessio Figalli. Regularity of optimal transport maps (after MaTrudinger-Wang and Loeper). Astérisque, 332:61, 2009. Séminaire Bourbaki 2008-2009, Exposé 1009.

[28] Alessio Figalli. Review of Cédric Villani's book "Optimal transport. Old and new". BAMS (NS), 47(4):723-727, 2010.

[29] Alessio Figalli. Stability in geometric \& functional inequalities. In European Congress of Mathematics Kraków, 2-7 July, 2012, pp. 585-599. EMS, 2014.

[30] Alessio Figalli. The Monge-Ampère equation and its applications. Zurich Lectures in Advanced Mathematics. European Mathematical Society, 2017. $x+200$ pages.

[31] Alessio Figalli. Lectio HC, 2019. https://www.upc.edu/ca/la-upc/ discursos-honoris/laudatio-discurs-figalli-eng.pdf, p. 6-9.

[32] Alessio Figalli and Laurent Desvillettes. Cédric Villani reçoit le prix de la Société Mathématique Européenne. Gazette SMF, 2009. 7 pages.

[33] Alessio Figalli and Grégoire Loeper. $C^{1}$ regularity of solutions of the Monge-Ampère equation for optimal transport in dimension two. Calculus of Variations and Partial Differential Equations, 35(4):537-550, 2009

[34] Alessio Figalli, Francesco Maggi, and Aldo Pratelli. A mass transportation approach to quantitative isoperimetric inequalities. Inventiones mathematicae, 182(1):167-211, 2010.

[35] Alessio Figalli and Ludovic Rifford. Continuity of optimal transport maps and convexity of injectivity domains on small deformations of $S^{2}$. Communications on Pure and Applied Mathematics, 62(12):1670-1706, 2009.

[36] Alessio Figalli, Ludovic Rifford, and Cédric Villani. On the MaTrudinger-Wang curvature on surfaces. Calculus of Variations and Partial Differential Equations, 39(3-4):307-332, 2010.

[37] Alessio Figalli, Ludovic Rifford, and Cédric Villani. Necessary and sufficient conditions for continuity of optimal transport maps on Riemannian manifolds. Tohoku Mathematical Journal, Second Series, 63(4):855-876, 2011.
[38] Alessio Figalli, Ludovic Rifford, and Cédric Villani. Nearly round spheres look convex. American Journal of Mathematics, 134(1):109-139, 2012.

[39] Alessio Figalli, Xavier Ros-Oton, and Joaquim Serra. Generic regularity of free boundaries for the obstacle problem. Publications Mathématiques IHÉS, pp. 1-112, 2020. https://arxiv.org/ pdf/1912.00714.pdf.

[40] J. Franch and S. Xambó-Descamps. Alessio Figalli, DHC of the UPC, 2019. https://fme.upc.edu/ca/la-facultat/activitats/2019-2020/ arxius/expo_alessiofigalli-dhc.pdf.

[41] Cristian E. Gutiérrez. The Monge-Ampère equation, volume 44 of Progress in Nonlinear Differential Equations and their Applications. Birkhäuser, 2001.

[42] Kevin Hartnett. A Traveller Who Finds Stability in the Natural World. Quanta Magazine, August 1st, 2018.

[43] Grégoire Loeper. On the regularity of solutions of optimal transportation problems. Acta mathematica, 202(2):241-283, 2009.

[44] Errico Presutti. Scaling limits in statistical mechanics and microstructures in continuum mechanics. Springer, 2008.

[45] Helga Rietz. Interview, 2018. Neue Zürcher Zeitung.

[46] Cédric Villani. Topics in optimal transportation. Number 58 in Graduate Studies in Mathematics. AMS, 2003. xvi+370 pages.

[47] Cédric Villani. Optimal transport: Old and new, volume 338 of Grundlehren der Mathematischen Wissenschaften. Springer, 2008. xxii+973 pages.

[48] Sebastià Xambó-Descamps. Alessio Figalli - Magia, método, misión. Boletín de la SEMA, 24 (April):12-29, 2020.

[49] Sebastià Xambó-Descamps. Alessio Figalli, DHC per la UPC. SCM/Notícies, 46(1):12, 2020.

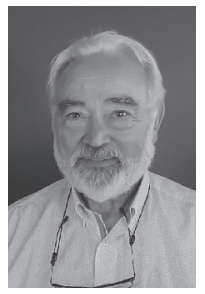

Sebastià Xambó-Descamps [https://mat. upc.edu/en/people/sebastia.xambo/] is an Emeritus Full Professor of the Universitat Politècnica de Catalunya at the Mathematics Department and currently is visiting the High Performance AI unit of the Barcelona Supercomputing Center. In 2019 he was awarded the Medal of the Real Sociedad

Matemática Española and in 2020 the Narcís Monturiol Medal of the Catalan Government. Author of Real Spinorial Groups - A Short Mathematical Introduction (2018). 